\title{
Evaluación de la capacidad antioxidante y actividad antibacteriana del extracto acuoso y etanólico de Cymbopogon citratus
}

\section{Evaluation of the antioxidante capacity and antibacterial activity of the aqueous anda ethanol extract of Cymbopogon citratus}

\author{
Henner Huamán Culqui ${ }^{1}$, César Rafael Balcazar Zumaeta ${ }^{2}$, Segundo Grimaldo Chávez Quintana ${ }^{3}$ Erick Aldo \\ Auquiñivin Silva ${ }^{4}$
}

\section{RESUMEN}

Se evaluó la capacidad antioxidante y actividad antibacteriana del extracto acuoso y etanólico de hierba luisa (Cymbopogon citratus), recolectada de doce distritos de la provincia de Bongará, región Amazonas. Se determinó mediante la técnica de DPPH que el extracto etanólico posee una mayor inhibición de radicales libres $(88,9 \pm 0,66 \%)$ en comparación con el extracto acuoso que presentó una actividad antioxidante en promedio de $82, .4 \pm 0,86 \%$. el extracto acuoso no logró tener efecto antimicrobiano sobre Escherichia coli, y un efecto moderado sobre Staphylococcus aureus; Se concluye que el etanol es el solvente que logra extraer un mayor contenido de metabolitos secundarios que repercute en su porcentaje de inhibición de radicales libres, de otro lado la actividad antibacteriana para extractos de hierba luisa aumenta en relación a la concentración del mismo.

Palabras clave: Actividad antimicrobiana, antioxidante, Cymbopogon citratus.

\begin{abstract}
The antioxidant capacity and antibacterial activity of the aqueous and ethanolic extract of lemongrass (Cymbopogon citratus), collected from twelve districts of the province of Bongará, Amazonas region, was evaluated. It was determined by the DPPH technique that the ethanol extract has a greater inhibition of free radicals $(88.9 \pm 0.66 \%)$ compared to the aqueous extract that presented an average antioxidant activity of $82.4 \pm$ $0.86 \%$ The ethanolic extract has a greater inhibition of free radicals $(88,9 \%)$ compared to the aqueous extract that presented an average antioxidant activity of $82,4 \%$. The aqueous extract failed to have an antimicrobial effect on Escherichia coli, and a moderate effect on Staphylococcus aureus; It is concluded that ethanol is the solvent that manages to extract a higher content of secondary metabolites that affects its percentage of free radical inhibition, on the other hand the antibacterial activity for luisa herb extracts increases in relation to its concentration.
\end{abstract}

Keywords: Antimicrobial activity, antioxidant, Cymbopogon citratus.

\footnotetext{
${ }^{1}$ Bachiller en Ingeniería Agroindustrial de la Facultad de Ingeniería y Ciencias Agrarias de la Universidad Nacional Toribio Rodríguez de Mendoza de Amazonas. Correo electrónico: hennerhc@gmail.com

${ }^{2}$ Proyecto SNIP N ${ }^{\circ} 381743$ - PROALIMENTOS y Docente de la Facultad de Ingeniería Ciencias Agrarias de la Universidad Nacional Toribio Rodríguez de Mendoza de Amazonas; Ingeniero Agroindustrial. Correo electrónico: cesar.balcazar@untrm.edu.pe

${ }^{3}$ Docente de la Facultad de Ingeniería Ciencias Agrarias de la Universidad Nacional Toribio Rodríguez de Mendoza de Amazonas; Ingeniero Agroindustrial; con el grado de Master en Economía Agroalimentaria y del Medio Ambiente. Correo electrónico: segundo.quintana@untrm ${ }^{\text {edu.p }}$

${ }^{4}$ Docente Asociado de la Facultad de Ingeniería Ciencias Agrarias de la Universidad Nacional Toribio Rodríguez de Mendoza de Amazonas; Ingeniero Agroindustrial; con el grado de Magíster en Ciencias de la Educación. Correo electrónico: erick.auquinivin@untrm.edu.pe
} 


\section{INTRODUCCIÓN}

Las plantas aromáticas en la región se han empleado por años como medicina natural debido a que estas plantas poseen antioxidantes naturales que actúan como protectores para la salud humana, sobre todo en la prevención de enfermedades. La presencia de antioxidantes en la agroindustria puede retrasar la oxidación de lípidos y una actividad antimicrobiana que es poco conocida en la población, generan un desaprovechamiento de sus beneficios.

Dentro de estas plantas, la Cymbopogon ymbopogon citratus comúnmente llamado hierba luisa pertenece a la familia poaceae, la cual es una planta tropical, cultivada como ornamental en muchas áreas templadas con una floración blanquecina (Nwachukwu et al., 2008)

La C. citratus se caracteriza por poseer hojas aromáticas con un olor similar al limón, y que se emplea en la medicina natural. Soto et al. (2017) y Vélez et al. (2018) ; mencionan que la hierba luisa es usada como infusión por sus para aliviar diferentes afecciones, como cólicos y otras dolencias estomacales, resfriados, fiebre y para calmar el dolor; y el contenido en vitamina $\mathrm{C}$ en esta planta lo constituye como una fuente de antioxidantes, con una importante actividad antibacteriana y antiinflamatoria.

La investigación abarcó la importancia del uso de extractos en la hierba luisa, debido a que los solventes interactúan sobre los compuestos bioactivos (metabolitos secundarios) (Echevarría et al., 2016). Esta dilución de metabolitos secundarios, permitirá estudiar la capacidad antioxidante y la actividad antimicrobiana de la hierba luisa frente a microorganismos patógenos.

Cheel et al. (2005) y Olorunnisola et al. (2014), indican que que en extractos se han identificado potenciales antioxidantes al demostrar una alta capacidad de decolorar el DPPH, y que los diferentes extractos de C. citratus poseen una captación de radicales libres con valores que oscilan entre $40 \mathrm{y}$ $68 \%$.

De otro lado, Soares et al. (2013) que el extracto etanólico de hierba luisa presenta una alta capacidad antioxidante. Asimismo, el extracto acuoso muestra menor poder reductor; y el extracto metanólico, aunque a menudo se lo conoce como el mejor solvente para extraer antioxidantes compuestos, determinaron una eliminación de radicales más bajo.

Fagbemi et al. (2009) y Olorunnisola et al. (2014), referente a la actividad antibacteriana de $C$. citratus indican que la presencia de los componentes $\alpha$-citral (geranial) y $\beta$-citral (neral) afectan su actividad antibacteriana para inhibir a bacterias Gram positivas y Gram negativas; $S$. aureus se inhibe a altas concentraciones de extracto acuoso y en el caso del extracto etanólico tuvo un buen efecto bactericida sobre $E$. coli.

Azuero (2016) y Okigbo y Mmeka (2008) encontraron que el extracto etanólico y acuoso de $C$. citratus existe inhibición sobre E. coli y S. aureus, con un diámetro de zona de inhibición de 16 y $10 \mathrm{~mm}$ respectivamente; ambos estudios demuestran que pueden utilizarse para tratamientos de afecciones intestinales, así como también en el tratamiento de procesos respiratorios.

La capacidad antioxidante y el estudio del efecto antibacteriano de las hojas de C. citratus fue el objetivo de esta investigación.

\section{MATERIAL Y MÉTODO}

Material: La muestra estuvo conformada por $300 \mathrm{~g}$ de hojas de hierba luisa que fueron recolectadas para cada distrito de la provincia de Bongará, región Amazonas.

Tabla 1. Distritos de la provincia de Bongará

\begin{tabular}{l|c}
\hline \multicolumn{1}{c|}{ Distritos } & Altitudes (msnm) \\
\hline Jazán & 1299 \\
\hline Churuja & 1383 \\
\hline San Carlos & 1890 \\
\hline Cuispes & 1937 \\
\hline Valera & 1978 \\
\hline Jumbilla & 1991 \\
\hline Yambrasbamba & 1995 \\
\hline Chisquilla & 2013 \\
\hline Shipasbamba & 2083 \\
\hline Recta & 2140 \\
\hline Corosha & 2180 \\
\hline Florida & 2225 \\
\hline
\end{tabular}

Después de la recolección de las muestras, estas fueron lavadas con agua destilada.

Preparación de extracto acuoso: Se utilizó lo descrito por Rangel et al. (2001) modificado, se colocó al fuego un recipiente con 01 litro de agua hasta su punto de ebullición, luego se apagó el fuego y se agregó las hojas de hierba luisa (200 gramos). Luego se cubrió el recipiente hasta su enfriamiento.

Preparación de extracto etanólico: Se utilizó la 
técnica descrita por Echevarría et al. (2016) modificada; en la cual se lavó las hojas de las plantas con agua destilada y fueron colocadas a temperatura ambiente. Posteriormente fueron llevadas a la estufa a $37^{\circ} \mathrm{C}$; luego se procedió a pesar 20 gramos de material vegetal molido y se adicionó $100 \mathrm{ml}$ de etanol al $96 \%$

Los extractos se maceraron por un tiempo de 48 horas para ser luego filtrados al vacío.

Evaluación de la capacidad antioxidante: Fue determinada siguiendo el método desarrollado por Brand-Williams (1995), modificado según cuyo tomado de Castañeda, Ramos e Ibañez (2008).

Preparación de las concentraciones de los extractos para la actividad antibacteriana: Se utilizó la técnica descrita por Merchán (2018), para un volumen inicial de extracto de $100 \mathrm{ml}$. Para esto se aplicará la siguiente fórmula:

$$
C 1 * V 1=C 2 * V 2
$$

Despejando V1, se obtiene: $V 1=\frac{C 2 * V 2}{C 1}$

Donde: C1: 100\% de la concentración inicial; C2: concentración a la que queremos llegar $(25,50,75 \mathrm{y}$ 100\%); V1: cantidad de $\mathrm{ml}$ de alcohol que debemos añadir para las concentraciones; V2: Cantidad inicial en $\mathrm{ml},(100 \mathrm{ml})$

Actividad antibacteriana: Se probaron los extractos de C. citratus frente a bacterias Escherichia coli y Staphylococcus aureus. Se empleó el método de difusión en discos desarrollado por Bauer et al. (1966), modificado por Picazo (2000) y Taroco et al. (2014):

Se depositó, en la superficie de agar de una placa previamente inoculada con el microorganismo. También Se preparó discos de papel secante impregnados con los diferentes extractos a diferentes concentraciones. Inmediatamente el disco impregnado se colocó en contacto con la superficie húmeda del agar, en donde el filtro absorbió el agua y el extracto difunde al agar.

Se difundió radialmente a través del espesor del agar a partir del disco formándose un gradiente de concentración. Transcurridas 18-24 horas de incubación los discos estuvieron estar rodeados por una zona de inhibición. En la presente investigación se mostrará el halo que se forma descontando la medida del disco inicial de inhibición (6 mm). Para ello se recurrió a lo indicado por (Calvo et al., 2006):

Valor de inhibición $(\mathrm{mm})=\frac{\emptyset_{\text {inhibición }}(\mathrm{mm})-\emptyset_{\text {disco }}(\mathrm{mm})}{2}$

Análisis de datos: Para el análisis de datos, estos fueron sometidos al análisis de varianza, e interacción para evaluar el factor altitud. Las diferencias se consideraron estadísticamente significativas con un nivel de $\mathrm{p}<0,05$.

\section{RESULTADOS}

Capacidad antioxidante de extracto etanólico y acuoso de C. citratus.

Tabla 2. Capacidad antioxidanteinhibición $( \pm$ d.e. $)$ mediante la técnica de DPPH

\begin{tabular}{c|lc|lc}
\hline $\begin{array}{c}\text { Altitud } \\
\text { msnm }\end{array}$ & \multicolumn{2}{|c|}{ \% Inhibición ( \pm d.e.) } & \multicolumn{2}{|c}{ \% Inhibición ( \pm d.e.) } \\
\hline $\mathbf{1 2 9 9}$ & EA & $82.40 \pm 0.86$ & EE & $87.62 \pm 0.59$ \\
\hline $\mathbf{1 3 8 3}$ & EA & $80.08 \pm 2.09$ & EE & $88.90 \pm 0.66$ \\
\hline $\mathbf{1 8 9 0}$ & EA & $79.80 \pm 1.00$ & EE & $86.35 \pm 1.10$ \\
\hline $\mathbf{1 9 3 7}$ & EA & $75.41 \pm 0.93$ & EE & $85.91 \pm 0.77$ \\
\hline $\mathbf{1 9 7 8}$ & EA & $80.40 \pm 1.18$ & EE & $86.95 \pm 1.14$ \\
\hline $\mathbf{1 9 9 1}$ & EA & $79.84 \pm 0.59$ & EE & $86.55 \pm 0.73$ \\
\hline $\mathbf{1 9 9 5}$ & EA & $75.57 \pm 1.06$ & EE & $87.03 \pm 0.73$ \\
\hline $\mathbf{2 0 1 3}$ & EA & $80.80 \pm 1.11$ & EE & $86.47 \pm 1.02$ \\
\hline $\mathbf{2 0 8 3}$ & EA & $75.77 \pm 0.82$ & EE & $86.51 \pm 0.73$ \\
\hline $\mathbf{2 1 4 0}$ & EA & $75.25 \pm 0.61$ & EE & $86.91 \pm 0.07$ \\
\hline $\mathbf{2 1 8 0}$ & EA & $77.52 \pm 0.25$ & EE & $86.95 \pm 0.52$ \\
\hline $\mathbf{2 2 2 5}$ & EA & $78.40 \pm 0.60$ & EE & $86.75 \pm 0.68$ \\
\hline
\end{tabular}

EA: Extracto acuoso; EE: Extracto etanólico

En la tabla 2 se observa el porcentaje de inhibición obtenido en los extractos estudiados; se observa que los mayores valores son de 88,9 y $82,4 \%$ en el extracto etanólico y acuoso respectivamente, asimismo se observa que el extracto etanólico presenta un mayor valor.

En la figura se observa que la altitud, el tipo de extractos y la interacción de extracto y altitud tuvieron efecto en el contenido del porcentaje de inhibición debido a que hay diferencias significativas del extracto y altitud sobre la capacidad antioxidante $(\mathrm{p}<0,05)$.

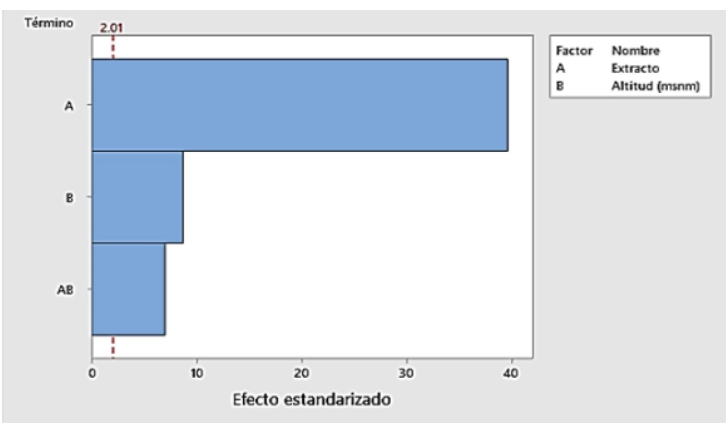

Figura 1. Diagrama de Pareto de la capacidad antioxidante (\% inhibición) 


\section{Actividad antibacteriana}

Los extractos etanólico y acuoso de hierba luisa presentan propiedades antimicrobianas tienen inhibición en S. aureus (gram + ) y E. coli (gram -). Observando que las muestras testigos $(0 \%$ de concentración) no demuestran tener efecto antimicrobiano sobre los organismos salvo un efecto leve en el extracto etanólico en S. aureus; asimismo se observa que existe mayor inhibición del extracto etanólico sobre ambas cepas.

Tabla 3. Actividades antimicrobianas de extracto acuoso y etanólicos de Cymbopogon citra. citratus sobre Escherichia coli y Staphylococcus aureus según altitud.

\begin{tabular}{|c|c|c|c|c|c|c|c|c|c|c|}
\hline \multicolumn{11}{|c|}{ Extracto etanólico } \\
\hline \multirow{3}{*}{ 异导 } & \multicolumn{5}{|c|}{ E.coli } & \multicolumn{5}{|c|}{ S. aureus } \\
\hline & \multicolumn{10}{|c|}{ \% Concentración } \\
\hline & 100 & 75 & 50 & 25 & $\mathbf{0}$ & 100 & 75 & 50 & 25 & $\mathbf{0}$ \\
\hline 1299 & + & + & + & + & - & + & + & + & + & - \\
\hline 1383 & + & + & + & + & - & + & + & - & + & - \\
\hline 1890 & - & + & - & + & - & + & + & - & - & - \\
\hline 1937 & + & + & + & + & - & + & + & + & - & + \\
\hline 1978 & + & + & - & - & - & - & - & + & - & - \\
\hline 1991 & + & + & - & - & - & + & - & - & + & - \\
\hline 1995 & + & + & + & - & - & + & - & + & - & - \\
\hline 2013 & - & + & - & - & - & + & + & + & - & - \\
\hline 2083 & + & - & - & + & - & + & - & - & + & + \\
\hline 2140 & + & - & - & - & - & + & + & - & - & - \\
\hline 2180 & + & + & + & + & - & + & + & + & + & - \\
\hline 2225 & + & + & + & + & - & + & + & + & + & - \\
\hline \multicolumn{11}{|c|}{ Extracto acuoso } \\
\hline \multirow{3}{*}{ 异总 } & \multicolumn{5}{|c|}{ E.coli } & \multicolumn{5}{|c|}{ S. aureus } \\
\hline & \multicolumn{10}{|c|}{ \% Concentración } \\
\hline & 100 & 75 & 50 & 25 & $\mathbf{0}$ & 100 & 75 & 50 & 25 & $\mathbf{0}$ \\
\hline 1299 & - & - & - & - & - & + & + & + & + & - \\
\hline 1383 & - & - & - & - & - & - & - & - & - & - \\
\hline 1890 & - & - & - & - & - & + & - & - & - & - \\
\hline 1937 & + & + & - & - & - & - & + & - & - & - \\
\hline 1978 & + & + & - & - & - & + & - & + & + & - \\
\hline 1991 & - & - & - & - & - & - & - & - & - & - \\
\hline 1995 & - & - & + & + & - & - & + & - & - & - \\
\hline 2013 & - & - & + & - & - & - & - & + & - & - \\
\hline 2083 & - & - & + & + & - & - & + & - & - & - \\
\hline 2140 & - & - & - & - & - & + & + & + & - & - \\
\hline 2180 & - & - & - & - & - & + & + & + & + & - \\
\hline 2225 & - & - & - & - & - & + & + & + & + & - \\
\hline
\end{tabular}

En la tabla 3 se observa que el extracto acuoso no logra tener efecto antimicrobiano sobre la cepa $E$. coli, pero si sobre S. aureus cuando las muestras proceden de una altitud baja y alta.

Tabla 4. Halo promedio de inhibición $(\mathrm{mm})$ de extractos de Cymbopogon citratus.

\begin{tabular}{|c|c|c|c|c|c|c|c|c|}
\hline & & & Extra & to eta & ólico & & & \\
\hline$=\overparen{\nexists}$ & & & oli & & & S. $a t$ & reus & \\
\hline 豆 & & & & Conc & ntraci & & & \\
\hline 《主 & 100 & 75 & 50 & 25 & 100 & 75 & 50 & 25 \\
\hline 1299 & 0.50 & 0.67 & 0.33 & 0.67 & 1.00 & 0.33 & 0.17 & 0.67 \\
\hline 1383 & 0.50 & 0.17 & 0.17 & 0.33 & 0.67 & 0.17 & 0.00 & 0.17 \\
\hline 1890 & 0.00 & 0.17 & 0.00 & 0.17 & 0.33 & 0.17 & 0.00 & 0.00 \\
\hline 1937 & 0.17 & 0.17 & 0.17 & 0.17 & 0.33 & 0.17 & 0.17 & 0.00 \\
\hline 1978 & 0.33 & 0.17 & 0.00 & 0.00 & 0.00 & 0.00 & 0.33 & 0.00 \\
\hline 1991 & 0.17 & 0.17 & 0.00 & 0.00 & 0.17 & 0.00 & 0.00 & 0.17 \\
\hline 1995 & 0.33 & 0.17 & 0.17 & 0.00 & 0.17 & 0.00 & 0.17 & 0.00 \\
\hline 2013 & 0.00 & 0.17 & 0.00 & 0.00 & 0.17 & 0.33 & 0.17 & 0.00 \\
\hline 2083 & 0.17 & 0.00 & 0.00 & 0.33 & 0.50 & 0.00 & 0.00 & 0.50 \\
\hline 2140 & 0.17 & 0.00 & 0.00 & 0.00 & 0.17 & 0.17 & 0.00 & 0.00 \\
\hline 2180 & 0.50 & 0.33 & 0.83 & 0.17 & 0.67 & 0.50 & 0.17 & 0.50 \\
\hline 2225 & 0.50 & 0.67 & 1.50 & 0.83 & 1.17 & 0.83 & 1.00 & 0.50 \\
\hline & & & Extı & cto a & oso & & & \\
\hline$\Xi \overparen{\dot{\Xi}}$ & & & & & & S. $a t$ & reus & \\
\hline$\stackrel{\Xi}{\dot{B}}$ & & & & Conc & ntraci & & & \\
\hline ४刍 & 100 & 75 & 50 & 25 & 100 & 75 & 50 & 25 \\
\hline 1299 & 0.00 & 0.00 & 0.00 & 0.00 & 0.33 & 0.17 & 0.17 & 0.17 \\
\hline 1383 & 0.00 & 0.00 & 0.00 & 0.00 & 0.00 & 0.00 & 0.00 & 0.00 \\
\hline 1890 & 0.00 & 0.00 & 0.00 & 0.00 & 0.33 & 0.00 & 0.00 & 0.00 \\
\hline 1937 & 0.50 & 0.17 & 0.00 & 0.00 & 0.00 & 0.17 & 0.00 & 0.00 \\
\hline 1978 & 0.50 & 0.33 & 0.00 & 0.00 & 0.33 & 0.00 & 0.17 & 0.17 \\
\hline 1991 & 0.00 & 0.00 & 0.00 & 0.00 & 0.00 & 0.00 & 0.00 & 0.00 \\
\hline 1995 & 0.00 & 0.00 & 0.33 & 0.33 & 0.00 & 0.17 & 0.00 & 0.00 \\
\hline 2013 & 0.00 & 0.00 & 0.50 & 0.00 & 0.00 & 0.00 & 0.17 & 0.00 \\
\hline 2083 & 0.00 & 0.00 & 0.17 & 0.17 & 0.00 & 0.33 & 0.00 & 0.00 \\
\hline 2140 & 0.00 & 0.00 & 0.00 & 0.00 & 0.33 & 0.17 & 0.17 & 0.00 \\
\hline 2180 & 0.00 & 0.00 & 0.00 & 0.00 & 0.33 & 0.33 & 0.50 & 0.17 \\
\hline 2225 & 0.00 & 0.00 & 0.00 & 0.00 & 0.50 & 0.50 & 0.83 & 0.33 \\
\hline
\end{tabular}

Se observa en la tabla 4 que conforme aumenta la concentración del extracto este demuestra una mayor actividad antimicrobiana; de otro lado el efecto antimicrobiano empieza a partir de una concentración del $25 \%$ en la mayoría de los casos.

El agua estéril utilizada como control negativo en la mayoría de los casos no mostró ningún efecto sobre los microorganismos de prueba.

\section{DISCUSIÓN}

La evaluación de la capacidad antioxidante permitió conocer que mediante el extracto etanólico se logra 
una mayor inhibición de radicales libres $(88,9 \pm 0,66 \%)$ respecto al extracto acuoso $(82,4 \pm 0,86 \%)$ de hierba luisa, esta comparación tiene relación con lo mencionado por Soares et al. (2013) que mencionan que extracto etanólico de $C$. citratus presenta una capacidad antioxidante superior, con resultados significativamente más altos. Este resultado en el extracto etanólico se puede deber como reporto Méndez et al. (2011) que a través de este solvente se logra una mayor extracción del contenido de metabolitos secundarios en comparación a otros extractos.

Los valores de inhibición hallados son superiores a los obtenidos por Cheel et al. (2005) que indican que en diferentes extractos de C. citratus presentaron una captación de radicales libres con valores que oscilan entre 40 y $68 \%$; en la investigación la capacidad antioxidante estuvo en un rango de 75 a $89 \%$, esta variación puede compararse con lo realizado por Méndez et al. (2011) indicando que debido a un alto contenido de fitofenoles en extractos etanol y acuoso se demuestra una mayor estabilización capacidad del radical libre.

El tipo de extracto muestra que existe diferencia significativa en la capacidad antioxidante en la hierba luisa; este efecto puede deberse según lo reportado por Cheel et al., (2005) donde mencionan que si bien las infusiones de hierba luisa carecen de sustancias tóxicas presentan un débil efecto antiinflamatorio, mientras que el extracto etanólico se caracteriza por una importante presencia de componentes que pueden ser quimiopreventivos para el cáncer.

Asimismo, la figura indica que existe diferencia significativa de la altitud sobre la capacidad antioxidante, aunque en $C$. citratus no se ha determinado la actividad antioxidante en función de la altitud en la que crece, puede compararse con otros trabajos como los realizados para Fagopyrum esculentum (Alforfón) en el que si se han encontrado diferencia de la actividad antioxidante debido a que la altitud puede tener efecto sobre el contenido de ácidos fenólicos, y el ambiente de crecimiento puede influir en las propiedades antioxidantes (Guo et al., 2011).

Referente a la actividad antibacteriana, se puede determinar que los extractos etanólico y acuoso tienen inhibición en ambos microorganismos, esta actividad antibacteriana es la similar a la reportada por Okigbo y Mmeka (2008), donde igualmente se encontró poder antimicrobiano frente a la $E$. coli $y S$. aureus. De otro lado, los resultados demuestran que las muestras testigos no tienen efecto antimicrobiano.

Se observó que el extracto acuoso no logra tener efecto antimicrobiano sobre la cepa $E$. coli, pero si sobre $S$. aureus cuando las muestras proceden de una altitud baja y alta. Asimismo, existe mayor inhibición del extracto etanólico sobre ambas cepas respecto al extracto acuoso; coincidiendo con lo encontrado por Bussmann et al. (2010) y Manvitha y Bidya (2014), en donde se indica que los extractos etanólicos de las hojas de hierba luisa muestra propiedades antibacterianas potenciales contra $E$. coli y $S$. aureus, debido a la presencia de flavonoides y taninos responsables de la actividad, estos extractos etanólicos exhiben una actividad más fuerte y mucho espectro de acción más amplio.

De otro lado, conforme aumenta la concentración del extracto se observa una mayor actividad antimicrobiana; en los extractos acuosos se observa que a altas concentraciones $(>50 \%)$ demuestran tener un amplio radio de acción (halos de inhibición) resultados que guardan relación con lo obtenido por Fagbemi et al. (2009). De otro lado, el extracto etanólico demuestra tener un espectro de acción más amplio que el extracto acuoso con mínimo de concentración del extracto del $25 \%$ esto coincide con lo encontrado por Azuero et al. (2016), Fagbemi et al. (2009) y Vélez et al. (2018), estableciendo a su vez que en el caso del extracto etanólico tuvo un buen efecto bactericida sobre $E$. coli.

En cuanto a la concentración, se observa que con concentraciones de $50 \%$ en adelante se logra tener una mayor actividad antimicrobiana respecto a las cepas en el extracto etanólico esta tendencia se confirma con lo reportado por Calvo et al. (2006) y Vélez et al. (2018), estableciendo que conforme se aumenta la concentración del extracto etanólico de hierba luisa el espectro de acción antimicrobiana aumenta (6halos de inhibición en el extracto etanólico) con lo cual puede utilizarse para tratamientos de afecciones intestinales como la salmonelosis y el cólera, así como también en el tratamiento de procesos respiratorios causados por la bacteria S. aureus (Azuero et al., 2016).

\section{CONCLUSIONES}

El extracto acuoso y etanólico de hierba luisa poseen una capacidad antioxidante superior al 75\%, asimismo, el etanol es mejor solvente que el agua puesto que logra extraer un mayor contenido de metabolitos secundarios que repercute en su porcentaje de inhibición.

La altitud de cultivo determina la capacidad antioxidante, aunque ende los extractos de $C$. citratus.

La actividad antibacteriana para de extractos de $C$. citratus el extracto etanólico presenta un mayor espectro de acción antimicrobiana sobre $E$. coli $y S$. 
aureus en comparación del extracto acuoso.

\section{REFERENCIAS BIBLIOGRÁFICAS}

Azuero, A., Jaramillo-Jaramillo, C., San Martín, D., \& D'Armas, H. (2016). Análisis del efecto antimicrobiano de doce plantas medicinales de uso ancestral en Ecuador. Revista Ciencia UNEMI, 9(20), 11-18.

Bauer, A. W., Kirby, W. M. M., Sherris, J. C., \& Turck, M. (1966). Antibiotic Susceptibility Testing by a Standardized Single Disk Method. American Journal of Clinical Pathology, 45 (4_ts), $493-496$. https://doi.org/10.1093/ajcp/45.4_ts.493

Brand-Williams, W., Cuvelier, M. E., \& Berset, C. (1995). Use of a free radical method to evaluate antioxidant activity. LWT - Food Science and Technology, 28(1), 25-30. https://doi.org/10.1016/S00236438(95)80008-5

Bussmann, R. W., Malca-García, G., Glenn, A., Sharon, D., Chait, G., Díaz, D., ... Benito, M. (2010). Minimum inhibitory concentrations of medicinal plants used in Northern Peru as antibacterial remedies. Journal of Ethnopharmacology, 132(1), 101$1 \quad 0 \quad 8$ https://doi.org/10.1016/j.jep.2010.07.048

Calvo, M. A., Angulo, E., Costa-Batllori, P., Adelantado, C., \& Vicente, A. (2006). Natural Plant Extracts and Organic Acids: Synergism and Implication on Piglet's Intestinal Microbiota. Biotechnology, 5(2), $\begin{array}{lllllll}1 & 3 & 7 & - & 1 & 4 & 2\end{array}$. https://doi.org/10.3923/biotech.2006.137.1 42

Castañeda, C. B., Ramos, Ll. E., \& Ibáñez, V. L. (2008). Evaluación de la capacidad antioxidante de siete plantas medicinales peruanas. Revista Horizonte Médico, 8(1), 56-72.

Cheel, J., Theoduloz, C., Rodríguez, J., \& SchmedaHirschmann, G. (2005). Free Radical Scavengers and Antioxidants from Lemongrass ( Cymbopogon citratus (DC.) Stapf.). Journal of Agricultural and Food Chemistry, 53 ( 7), $2511-2517$. https://doi.org/10.1021/jf0479766

Echevarría, A., D’Armas, H., Matute-L., N.-L., Jaramillo, C., Rojas-de-Astudillo, L., \& Benítez, R. (2016). Evaluación de la capacidad antioxidante y metabolitos secundarios de extractos de dieciséis plantas medicinales. Revista Ciencia UNEMI, 9(20), 29-35.

Fagbemi, J., Ugoji, E., Adenipekun, T., \& Adelowotan, O. (2009). Evaluation of the antimicrobial properties of unripe banana (Musa sapientum L.), lemon grass (Cymbopogon citratus S.) and turmeric (Curcuma longa L.) on pathogens. African Journal of Biotechnology, 8(7), 1176-1182.

Guo, X.-D., Ma, Y.-J., Parry, J., Gao, J.-M., Yu, L.-L., \& Wang, M. (2011). Phenolics Content and Antioxidant Activity of Tartary Buckwheat from Different Locations. Molecules, 16(12), $\begin{array}{lllllllll}9 & 8 & 5 & 0 & - & 9 & 8 & 6 & 7\end{array}$. https://doi.org/10.3390/molecules 16129850

Manvitha, K., \& Bidya, B. (2014). Review on pharmacological activity of Cymbopogon citratus. International Journal of Herbal Medicine, 1(6), 5-7.

Méndez, J. J., Murillo, E., Yara, E., Suescún, W. F., Osorio, J. N., \& Mosquera, M. (2011). Climate influence on chemical composition and antioxidant activity of Justicia pectoralis Jacq. Revista Cubana de Farmacia, 45(1), 88-100.

Merchán, M. S. (2018). Evaluación de la actividad antibacteriana de extracto alcohólico y extracto etéreo de Annona muricata frente a Pseudomona aeruginosa. Universidad Regional Autónoma de los Andes, 1(1).

Nwachukwu, I., Allison, L. N., Chinakwe, E., \& Nwadiaro, P. (2008). Studies on the effects of Cymbopogon Citratus, Ceiba Pentandra and Loranthus Bengwelensis extracts on species of dermatophytes. J Am Sci, 4, 1545-1603.

Okigbo, R., \& Mmeka, E. (2008). Antimicrobial Effects Of Three Tropical Plant Extracts On $<I>$ Staphylococcus aureus, Escherichia coli and Candida albicans $</ I>$. African Journal of Traditional, Complementary and Alternative Medicines, 5(3), 226-229. https://doi.org/10.4314/ajtcam.v5i3.31277

Olorunnisola, S. K., Asiyanbi, H. T., Hammed, A. M., \& Simsek, S. (2014). Biological properties of lemongrass: An overview. International Food Research Journal, 21(2), 455-462.

Picazo, J. (2000). Métodos básicos para el estudio de la sensibilidad a los antimicrobianos. Procedimientos en Microbiología, 11. 
Rangel, D., Garcia, I., Velasco, J., \& Buitrago, D. (2001). Actividad antimicrobiana de los extractos etanólico, acetónico y acuoso de Baccharis nitida (Ruiz et Pavon) Pers. 42, 5.

Soares, M. O., Alves, R. C., Pires, P. C., Oliveira, M. B. P. P., \& Vinha, A. F. (2013). Angolan Cymbopogon citratus used for therapeutic benefits: Nutritional composition and influence of solvents in phytochemicals content and antioxidant activity of leaf extracts. Food and Chemical Toxicology, 60, $\begin{array}{lllllll}4 & 1 & 3 & - & 4 & 1 & 8\end{array}$. https://doi.org/10.1016/j.fct.2013.07.064

Soto, M. R., Alvarado, P. A. A., Rosales, L. E., \& Cerna, J. (2017). Efecto del aceite esencial de Cymbopogon citratus (dc.) Stapf hierba luisa en los niveles de ansiedad de estudiantes de educación secundaria. In $\mathrm{C}$ r e s c e $\mathrm{n} \mathrm{d} \mathrm{o,} 8(1), 22$. https://doi.org/10.21895/incres.2017.v8n1. 03

Taroco, R., Seija, V., \& Vignoli, R. (2014). Métodos de estudio de la sensibilidad antibiótica. Temas de Bacteriología y Virología médica, 663-671.

Vélez, R., D’Armas, H., Jaramillo-Jaramillo, C., \& Vélez, E. (2018). Metabolitos secundarios, actividad antimicrobiana y letalidad de las hojas de Cymbopogon citratus (hierba luisa) y Melissa officinalis (toronjil). FACSaludUNEMI, 2(2), 31-39. 